\title{
INSEGURIDAD DE LA PRODUCCIÓN AGRÍCOLA DE ALIMENTOS POR EROSIÓN HÍDRICA EN LA CUENCA DEL RÍO SIGUAS - REGIÓN AREQUIPA
}

\author{
Insecurity of agricultural food production by water erosion: Siguas river basin - Arequipa \\ Eliana G. Contreras ${ }^{1}$, Marcelo Portuguez ${ }^{2}$, Ana M. Muñoz ${ }^{3}$, Martín Condorhuaman ${ }^{3}$ \\ ${ }^{1}$ Instituto de Investigación en Química Biológica, Microbiología y Biotecnología, "Marco Antonio Garrido Malo", Facultad de \\ Farmacia y Bioquímica, Universidad Nacional Mayor de San Marcos. ${ }^{2}$ Facultad de Ingeniería Agrícola, Universidad Nacional \\ Agraria La Molina. ${ }^{3}$ Instituto de Investigación en Ciencias Farmacéuticas y Recursos Naturales "Juan de Dios Guevara"- \\ Facultad de Farmacia y Bioquímica, Universidad Nacional Mayor de San Marcos
}

\section{RESUMEN}

En la presente investigación se estimó la pérdida de suelos por erosión hídrica, que es el principal problema ambiental que limita la producción de alimentos al influir negativamente en la capacidad del suelo para sostener una agricultura productiva, poniendo en riesgo a poblaciones rurales cuya economía y alimentación se basan en la producción agrícola de alimentos. En la estimación de pérdida de suelos se empleó la Ecuación Universal de Perdida de Suelos (USLE, por sus siglas en inglés) y una metodología basada en los Sistemas de Información Geográfica (SIG), los que generaron resultados en cantidad, intensidad y localización del fenómeno en estudio. Se identificaron suelos con erosión media, alta, muy alta y crítica en los distritos de Lluta y Huanca de la provincia de Caylloma, estudiándose la tendencia de la producción agrícola (12 años) de alfalfa, por ser el cultivo permanentemente empleado para alimentar al ganado vacuno con fines de producción lechera para abastecer la demanda de la empresa GLORIA S.A., y también se estudiaron los cultivos transitorios de trigo y papa. La producción agrícola de alfalfa mejora la fertilidad de los suelos, asimismo sirve como cobertura vegetal, lo que permite la recuperación de suelos erosionados, también la práctica conservacionista de rotación de cultivos permite que se mantenga la producción agrícola de los cultivos transitorios, considerados representativos para la seguridad alimentaria internacional.

Palabras clave: Erosión hídrica, Siguas, producción agrícola de alimentos.

\section{SUMMARY}

In present investigation was estimated the loss of soil by water erosion, which is the main environmental problem that limits food production because to affect adversely the ability of the soil to sustain productive agriculture, threatening to rural populations whose economy and nourishment are based on agricultural food production. To estimating soil loss, the Universal Soil Loss Equation (USLE for its acronym in English) and a methodology based on Geographic Information Systems (GIS) were used, which yielded results in quantity, intensity and location of the phenomenon. Were identified soils with medium, high, very high, and critical erosion_degree in the districts of Lluta and Huanca from Caylloma province, being studied the trend to produce alfalfa since 12 years ago because is the permanent crop employee to feed cattle milk producer to meet the demand of the company GLORIA S.A., and also were studied annual crops of wheat and potatoes. Agricultural production of alfalfa improving soil fertility, also serves as vegetable coverage it allows eroded soil recovery; as well the the conservation practice by crop rotation allow keeping those transients agricultural production crops: potato and wheat, considered representative yo food safety international.

Keywords: Water erosion, Siguas, agricultural food production.

\section{INTRODUCCIÓN}

$\mathrm{N}^{0}$ uestro planeta enfrenta varios desafíos trascendentes, entre estos la creciente demanda de alimentos como consecuencia del crecimiento demográfico. Actualmente se estima la población mundial alrededor de 7210 millones de personas, cifra que superará los 9000 millones para el año $2050^{(1)}$. La función de la agricultura es asegurar que distintos alimentos estén disponibles y accesibles en todo momento para satisfacer las necesidades de las poblaciones. Asimismo, las familias de agricultores gestionan los recursos agrícolas del mundo y suministran más del $80 \%$ de los alimentos; sin embargo, muchas de ellas son pobres y se encuentran en una situación de inseguridad alimentaria (FAO, 2014) ${ }^{(2)}$.

Esto motiva a considerar la producción agrícola de alimentos en áreas afectadas por el principal 
problema ambiental de los suelos en nuestro país, la erosión hídrica, que consiste en la pérdida gradual del material del suelo por la acción dispersante de las gotas de lluvia y por la escorrentía ${ }^{(3)}$, ocasionando que el suelo dañado pierda su capacidad productiva, con la consecuente reducción de los cultivos.

La cuenca del río Siguas está conformada por la sub cuenca Alto Siguas, cuyos suelos presentan relieves topográficos ligeramente inclinados $(2-10 \%)$, mayoritariamente superficiales y poco profundos, que han ido perdiendo su capa arable debido al sistema de riego, erosión ocasionada por las lluvias y su topografía; presentan ligera pedregosidad y buen contenido de materia orgánica. La sub cuenca Bajo Siguas presenta suelos cuyas pendientes fluctúan de o a $5 \%$, de textura predominantemente franco-arenosa, con alto contenido de materia orgánica. Los suelos ubicados en las partes bajas y colindantes con el río presentan problemas de drenaje y salinidad debido a las filtraciones provenientes de la irrigación Majes, por lo que en estas áreas sólo crecen pastos naturales ${ }^{(4)}$. En la parte media de la sub cuenca del río Siguas no hay quebradas, solo cárcavas productos de la erosión hídrica.

Hidrográficamente, el río Siguas se forma al confluir los ríos Lluta y Lihualla, y luego al unirse con el río Vitor forman el río Quilca, que desemboca al Océano Pacifico. Tiene como fuentes de alimentación los deshielos de los nevados Ampato y Sabancaya, así como parte del Hualca Hualca y Ananto, además de las precipitaciones pluviales de las partes altas de la cuenca ${ }^{(4)}$.

\section{MATERIAL Y MÉTODOS}

Es un estudio de tipo descriptivo, retrospectivo y transversal.

\section{Material:}

- Información cartográfica: carta Nacional (escala: 1/100ooo) del Instituto Geográfico Nacional, mapa de ubicación espacial de las estaciones (escala: 1/100ooo) del SENAMHI, mapa de cobertura y uso (escala: 1/500oo) del INRENA, mapa de suelo (escala: 1/500oo) de la FAO.

- Información pluviográfica: precipitaciones media anuales (20 años de registro).

- Información estadística: producción agrícola (tn) de alfalfa, papa y trigo de los años 20002012 (Base de datos de la Gerencia Regional de Agricultura Arequipa) ${ }^{(5)}$.
Área de estudio: la cuenca del río Siguas se encuentra ubicada políticamente entre las provincias de Arequipa y Caylloma en la Región Arequipa - Perú (figura 1), coordenadas Este 148 ooo/ 215 ooo Norte 8157 500/ 8 258650 , entre 4670 y 150 metros de altitud, abarcando una extensión de $1774 \mathrm{~km}^{2}$.

\section{Metodología empleada:}

Determinación de la pérdida de suelo por erosión hídrica: se aplicó el método denominado Ecuación Universal de Pérdida de Suelos (USLE, por sus siglas en inglés) de Wischmeier y Smith ${ }^{(6)}$. La expresión más conocida de esta ecuación, es:

$$
\text { A = R.K.S.L.C.P }
$$

Donde: $\boldsymbol{A}$, es la pérdida de suelo por unidad de superficie (tn/ha.año). $\boldsymbol{R}$, es el factor lluvia o índice de erosión pluvial (MJ. mm/ha h). $\boldsymbol{K}$, es el factor erodabilidad del suelo (tn.ha.h/ha.MJ.mm). $L$, es el factor longitud de pendiente (adimensional). $\boldsymbol{S}$, es la pendiente (adimensional). $\boldsymbol{C}$, es el factor cultivo $\mathrm{y} / \mathrm{u}$ ordenación y $\boldsymbol{P}$, es el factor prácticas de cultivo.

Yataco (7) refiere que de los factores considerados en la USLE, $\mathrm{R}$ no puede ser modificado, $\mathrm{K}$ depende fuertemente de la textura de los suelos, la cual no puede ser fácilmente mejorada. Sólo C, P, y LS (el factor LS combinado o factor topográfico, representa la proporción de pérdida de suelo de una longitud e inclinación dada) podrían ser modificados; así, las prácticas agronómicas comunes pueden utilizarse para alterar la cobertura vegetal y consecuentemente $C$ mediante el manejo de desechos y mejoramiento de los sistemas de siembra. El factor $\mathrm{P}$ puede ser cambiado por prácticas agronómicas, como las barreras vegetativas, o con obras de conservación de suelos como terrazas, zanjas o acequias de ladera. La modificación del factor P está íntimamente ligado a la reducción de la longitud de pendiente, L. Por último, la pendiente $S$, sólo puede ser modificada mediante obras de conformación del terreno, tales como terrazas.

Factores de la Ecuación Universal de Pérdida del Suelo: El factor R se calculó empleando la ecuación recomendada por Roose (1995), citado por Ramos ${ }^{(8)}$, luego se generó el mapa de isoerodientes, del cual se obtuvo la superficie que representa el factor R; a partir del Modelo Digital de Elevación (MDE) se obtuvieron los mapas de pendientes y de direcciones que luego se relacionaron con los valores del factor LS asignados directamente a una ladera ${ }^{(8)}$; el factor $\mathrm{K}$ se obtuvo a partir del mapa geológico; el factor C (protección de la cobertura vegetal cuando intercepta las gotas de lluvia 


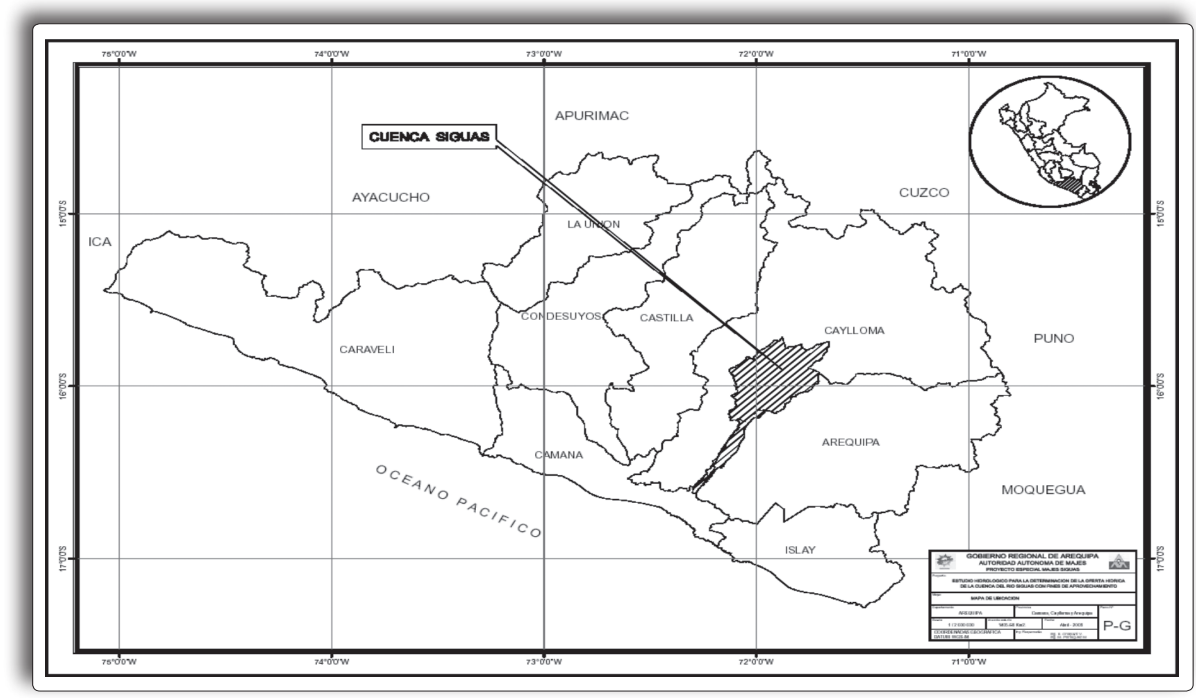

Figura 1. Mapa de ubicación de la cuenca del río Siguas.

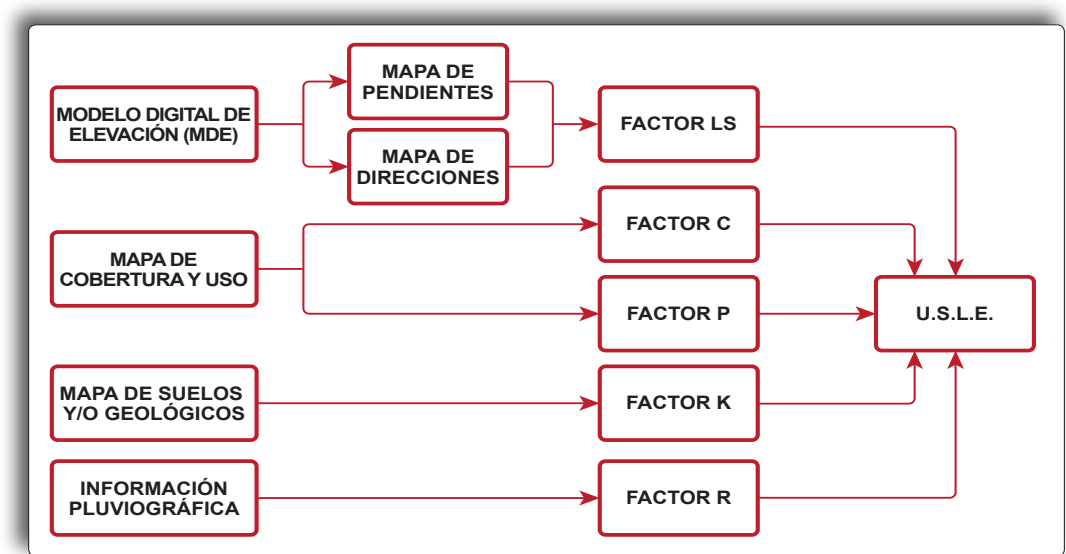

Figura 2. Modelo conceptual para la estimación de los niveles de pérdida de suelo según el método USLE.

amortiguando su energía de impacto disminuyendo el efecto erosivo), se determinó a partir del mapa temático de cobertura y los valores de la tabla publicada por Wischmeier y Smith ${ }^{(4)}$, quienes definen los valores de C para los tipos más comunes de cultivos, así como para las diferentes coberturas; el factor P se consideró igual a 1 , tal como lo recomienda Rodríguez ${ }^{(9)}$, cuando no es posible el conocimiento de estas prácticas de control de la erosión ni de manejo de las actividades productivas.

Estados erosivos: se elaboró el mapa de estados erosivos a partir de la clasificación de la FAO (1980) ${ }^{(10)}$, para la cuenca del río Siguas (Arequipa) (figura 3), superponiendo los mapas (análisis espacial) de los factores reseñados anteriormente $(K, L S, C, R)$ siguiendo el diseño del mapa conceptual que se muestra en la figura 2.

Producción agrícola de alimentos: se evaluó la tendencia de la producción agrícola de algunos cultivos alimenticios (12 años de registro) en los suelos clasificados con erosión alta hasta crítica.

\section{RESULTADOS}

La erosión hídrica de los suelos en la cuenca Siguas se clasifican en bajo, moderado, medio, alto, muy alto y crítico, donde $48,6 \%$ del total de la superficie de los suelos se clasifican con erosión baja y $1,58 \%$ suelos con erosión hídrica crítica.

La erosionabilidad del suelo predominante en la cuenca se relaciona a conglomerados y arenas aluviales (factor $\mathrm{K}=0,48$ t.ha.h/ha.MJ.mm), que son suelos moderadamente susceptibles al desprendimiento y a la erosión.

En la cuenca estudiada, la erosión va de media a crítica en los distritos de Huanca y Lluta de la provincia de Caylloma, lo que corresponde a pérdidas entre 600 a $1400 \mathrm{Tn} / \mathrm{ha} / \mathrm{año}$; en los distritos de Huambo y Cabanaconde de la provincia de Caylloma la erosión está entre moderada y media (figuras 4 y 5 ).

Los valores de precipitación promedio anual en el ámbito de la cuenca estuvo entre $1,8 \mathrm{~mm}$ y $710 \mathrm{~mm}$; en el distrito de Lluta (300o metros de altitud) la precipitación promedio anual fue $144,8 \mathrm{~mm}$, en Huanca (3080 metros de altitud) 110,0 mm.

En las figuras 6, 7 y 8 se observan las producciones agrícolas anuales de alfalfa, trigo y papa en los distritos de Lluta y Huanca de la provincia de Caylloma, durante los 12 años (2000-2012).

\section{DISCUSIÓN}

La aplicación de los SIG permiten la estimación cuantitativa de pérdida de suelos por erosión hídrica a fin de orientar la toma de decisiones políticas a gran escala (11-13), pues los resultados obtenidos indican dónde y en qué cantidad se produce el fenómeno erosivo, posibilitando localizar espacialmente el fenómeno y su intensidad (figuras 3, 4 y 5). Nuñez ${ }^{(14)}$, señala que el efecto erosivo de la gota de lluvia se magnifica cuando se conjugan otras variables como las 


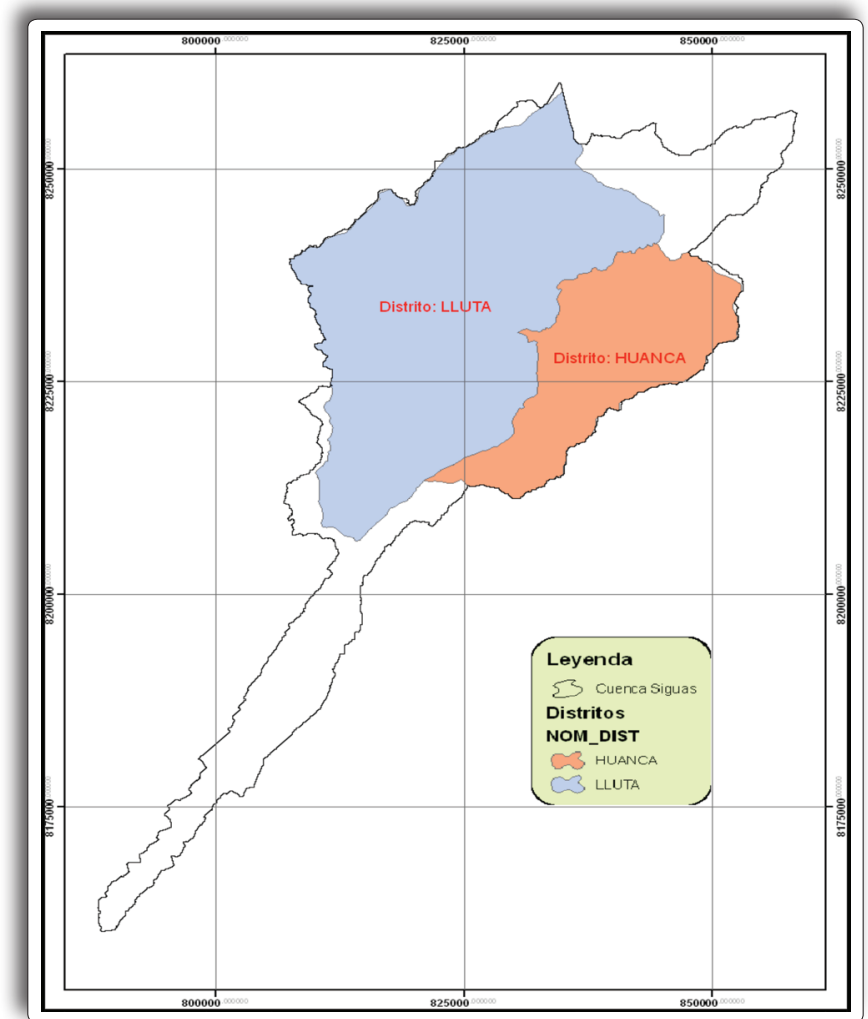

Figura 3. Ubicación de los distritos en la Cuenca Siguas.

pendientes abruptas, ausencia de cobertura vegetal, condición textural o el pobre desarrollo estructural de los suelos. La intensidad y prolongación de los eventos lluviosos son considerados principio del efecto erosivo de la lluvia; el régimen pluviométrico, que registran las lluvias en la zona de estudio, se caracteriza por su irregularidad temporaldemayor frecuenciaeintensidad entre los meses de diciembre a abril, mientras que el resto del año es baja, considerándose casi nula en los meses de junio a agosto; la precipitación promedio anual en el ámbito de la cuenca es considerada de baja erosividad ${ }^{(15,16)}$, lo que nos indica que el factor erosivo de la lluvia no sería causante de la erosión hídrica en la zona de estudio.

Las unidades litológicas obtenidas a partir de la digitalización de los mapas geológicos, permiten una reclasificación para identificar las mismas con su valor del factor $\mathrm{K}$ y obtener así la distribución espacial de la erodabilidad de los suelos ${ }^{(9)}$. Los valores del factor $\mathrm{K}$, nos revelan que en el ámbito de la cuenca Siguas los suelos corresponden a suelos moderadamente susceptibles al desprendimiento, siendo este un factor preponderante para la erosión hídrica.

El factor LS (factor topográfico) fue determinado al interpolar las isolineas del mapa topográfico por

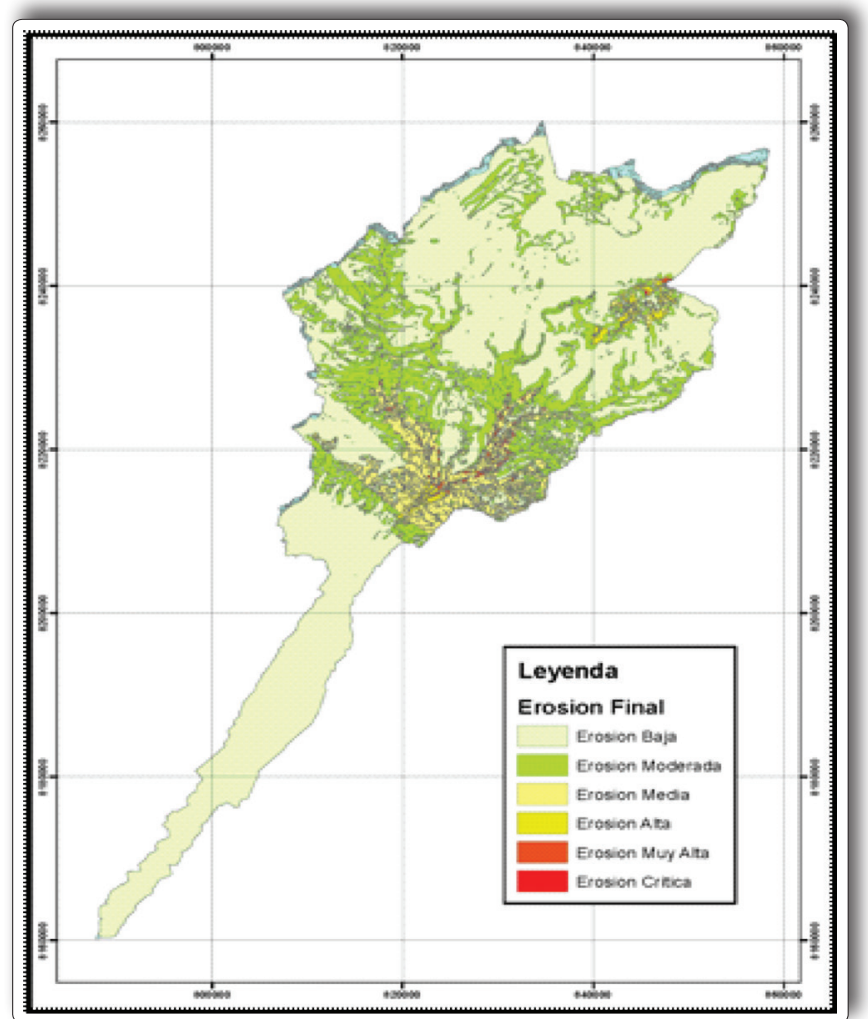

Figura 4. Mapa de estados erosivos en la Cuenca Siguas.

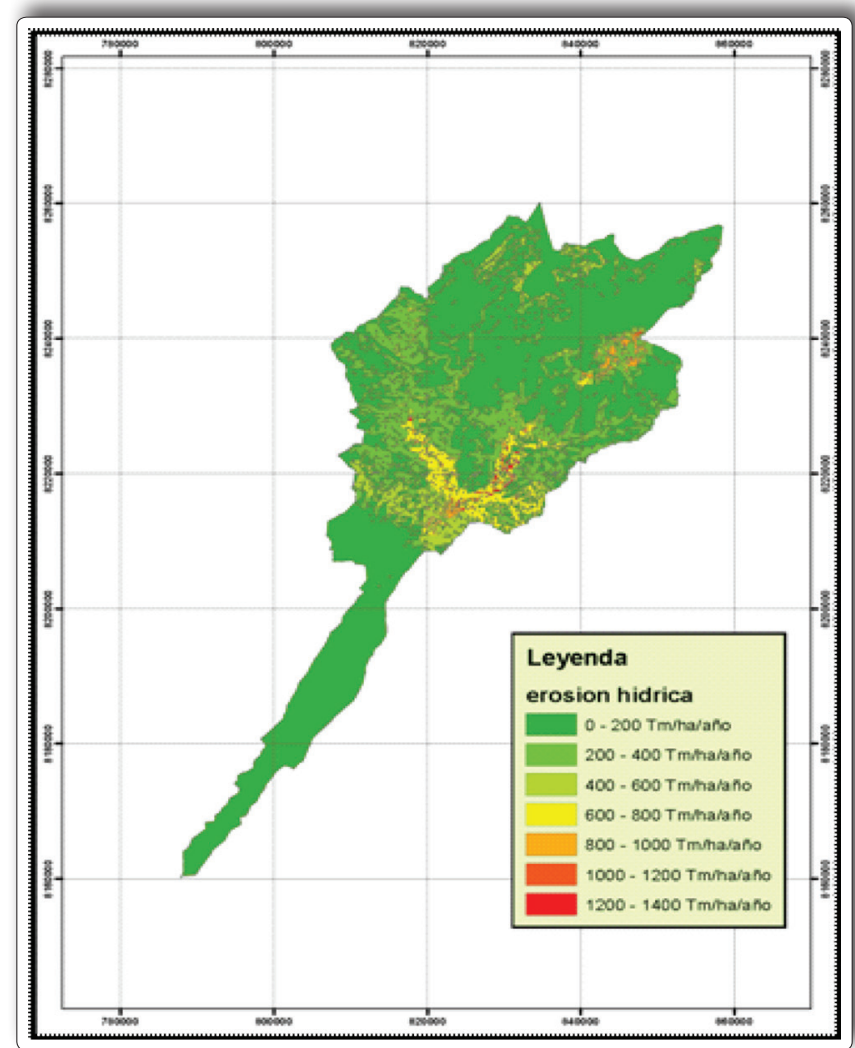

Figura 5. Mapa de pérdida de suelos por erosión hídrica en la cuenca Siguas. 
el método de triangulación, con el software Arc GIS obteniendoel Modelo Digital de Elevación (MDE) del que se obtuvo un mapa de pendientes (utilizando el módulo slope de Arc GIS) que refleja los distintos valores del factor S; por otra parte, el factor L se promedió para asignar a un gradiente de pendientes, la topografía de la zona propicia para la pérdida de suelos por erosión hídrica.

El factor C, referido a la protección que la cobertura vegetal proporciona al suelo al interceptar las

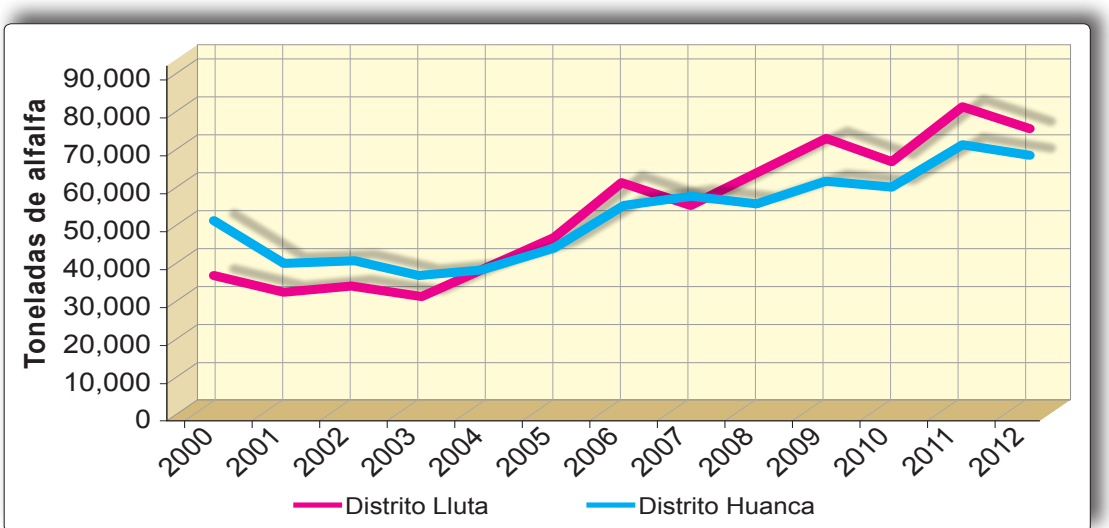

Figura 6. Producción agrícola de alfalfa en los distritos de Lluta y Huanca de la provincia de Caylloma - Región Arequipa. gotas de lluvia y amortiguar su energía de impacto y de escorrentía, disminuye el efecto erosivo. Los valores del factor $C$ se obtuvieron de los valores de la tabla para valores de este factor de Wischmeier y Smith ${ }^{(6)}$, consiguiéndose el mapa con la distribución espacial del factor $\mathrm{C}$ a partir del mapa de cobertura y uso.

Con las capas temáticas obtenidas, correspondientes a los factores del método USLE, se calcularon los valores de las tasas de erosión multiplicando los mapas correspondientes. Los mapas de estados erosivos y de pérdida de suelos para la zona en estudio muestran las tasas más altas de pérdida de suelo (figuras 4 y 5), correspondientes a superficies de topografía con pendientes abruptas, poca estructura de los suelos, textura arenosa y cobertura vegetal inapreciable.

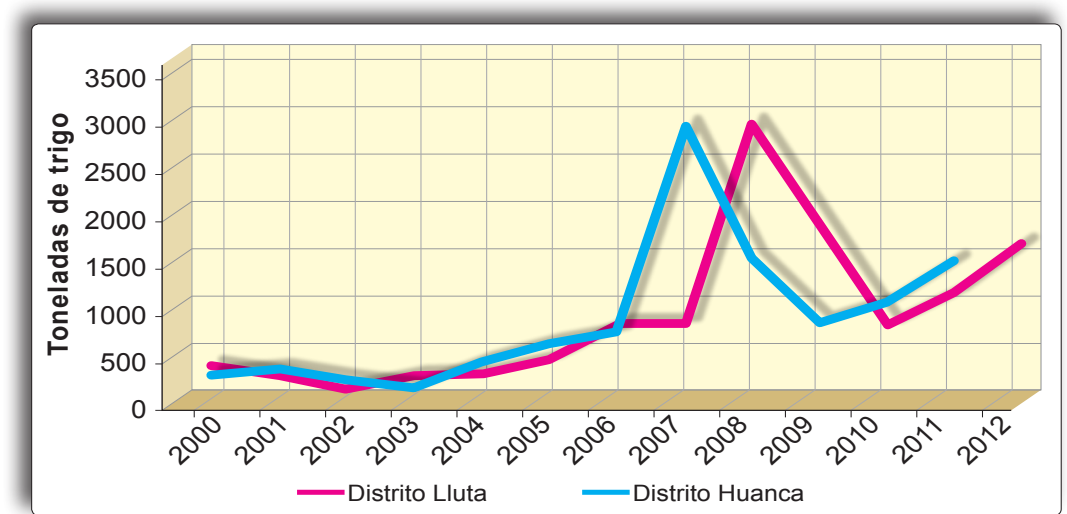

Figura 7. Producción agrícola de trigo en los distritos de Lluta y Huanca de la Provincia de Caylloma - Región Arequipa.

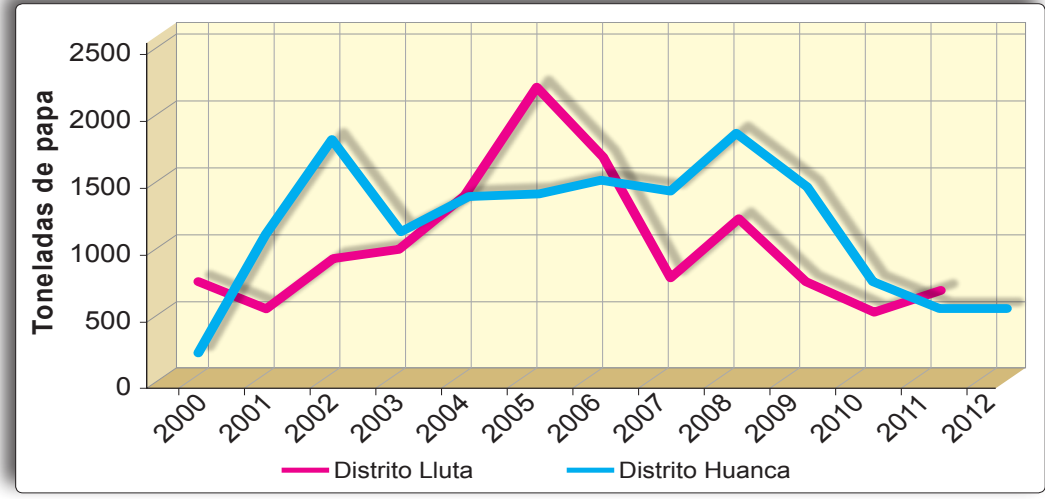

Figura 8. Producción agrícola de papa en los distritos de Lluta y Huanca de la Provincia de Caylloma - Región Arequipa.
El principal problema que limita la producción agrícola de alimentos es la erosión hídrica, por tal motivo evaluamos el comportamiento de la producción agrícola de algunos alimentos en los suelos de los distritos de Huanca y Lluta, por presentar mayores daños por erosión hídrica. El cultivo predominante en la zona de estudio es la alfalfa (Medicago sativa) que es el cultivo permanente. Golberg (17), refiere que el cultivo de alfalfa permite recuperar áreas con escasa fertilidad, consiguiendo incluso aumentar el rendimiento de trigo ya que las leguminosas proporcionan nitrógeno y contribuyen a la fertilidad del suelo ${ }^{(18)}$; además, la alfalfa es un cultivo forrajero que ofrece una buena protección al suelo y se emplea principalmente para alimentar al ganado vacuno. La producción de alfalfa (figura 6) en los últimos años va en aumento, dado que este cultivo permite la recuperación de la fertilidad de los suelos erosionados, en los distritos mencionados.

La producción agrícola de trigo (figura 7) tiene una tendencia irregular por ser un cultivo transitorio dada la 
rotación de cultivos como un método conservacionista de los suelos en los distritos de Lluta y Huanca. El trigo requiere suelos profundos_para el buen desarrollo del sistema radicular ${ }^{(19)}$, y es considerado como el cultivo más importante a nivel mundial para la seguridad alimentaria. Asimismo, la papa es el tercer cultivo más representativo para la seguridad alimentaria a nivel internacional y se le ha denominado como un "arma milenaria” capaz de enfrentar el hambre y la pobreza ${ }^{(20)}$; la producción agrícola de papa (figura 8) en los últimos años sigue un comportamiento irregular, dado que a mayor rendimiento del cultivo, es mayor a la extracción de nutrientes minerales del suelo, por lo que este es un cultivo transitorio en los distritos señalados.

\section{CONCLUSIONES}

- Los suelos con mayores tasas de erosión hídrica se ubican en los distritos Lluta y Huanca de la provincia de Caylloma - región Arequipa, lo que se evidencia por la presencia de cárcavas (erosión crítica).

- La producción agrícola de alfalfa permite la recuperación de suelos erosionados, mejora la fertilidad de los mismos y ayuda a sostener los cultivos de rotación o transitorios como son el trigo y la papa.

\section{REFERENCIAS BIBLIOGRÁFICAS}

1. Fundación Internacional de Desarrollo Agrícola. ¿Por qué el FIDA? Roma; 2014.

2. Organización delas Naciones Unidas para laAlimentación y la Agricultura. El estado mundial de la agricultura y la alimentación - La innovación en la agricultura familiar. [Internet]. FAO. Roma; 2015. [Citado 12 febrero 2015]. Disponible en: http://www.fao.org/3/a-i4040s.pdf

3. Porta J, López-Acevedo M, Roquero C. Edafología para la agricultura y el medio ambiente. $3^{\text {ra }}$ ed. Ediciones Mundi Prensa. Madrid; 2003.

4. Autoridad Nacional del Agua (ANA). Diagnóstico de los problemas y conflictos en la gestión del agua en la cuenca Chili-Quilca. Ministerio de Agricultura y Riego. Perú; 2008.

5. Gerencia Regional de Agricultura Arequipa. Estadísticas agrícolas. [En Línea] Acceso 12 set 2014. Disponible en http://www.agroarequipa.gob.pe/seriehist $\% \mathrm{C}_{3} \% \mathrm{~B}_{3}$ rica-de-campa $\% \mathrm{C}_{3} \% \mathrm{~B}_{1}$-agr $\% \mathrm{C}_{3} \%$ ADcolas

6. Wischmeier $\mathrm{WH}$, Smith DD. Predicting rainfall erosion losses. A guide to conservation planning. U.S.D.A. Agriculture Handbook 1978; 537: 1-49.

7. Yataco A. Evaluación cuantitativa de la erosión hídrica utilizando varillas de erosión en suelos con diferentes grados de cobertura vegetal de la comunidad campesina de Yuracmayo, Lima. [Tesis para obtener el grado de
Magister Scientae Especialidad en Ciencias Ambientales]. Escuela de Post Grado. Universidad Nacional Agraria La Molina. Lima, 2007.

8. Ramos C. Modelamiento Ambiental para análisis de susceptibilidad erosiva en la cuenca media y alta del río Cañete y determinación del mapa de erosión (Sistema de Información Geográfica). Facultad de Ingeniería Agrícola. UNALM. Lima; 2001.

9. Rodríguez M. Potencialidad de las técnicas SIG para la Gestión Medioambiental: Aplicación al estudio de la erosión. M+A. Revista Electrónica Medio Ambiente Mayo [Internet]. 2007 [Citado 3 dic 2010]; 3: 76-89. Disponible en http://www.ucm.es/info/iuca/2007\%20 1\%2oRODRIGUEZ.pdf.

10. Mármol LA. Introducción al manejo de cuencas hidrográficas y corrección de torrentes. Manejo de cuencas hidrográficas. Facultad de Ciencias Naturales. Universidad Nacional de Salta. Salta; 2006.

11. Gallego FJ, Cobo M, Navarrete L, Valderrama J, Jiménez R. Determinación de riesgos de erosión en la comarca olivarera de "Sierra Mágina" (Jaén) mediante técnicas SIG y Teledetección. Procedente de las Actas del XIV Congreso Internacional de Ingeniería; 2002 junio 5-7; Universidad de Cantabria, Santander: Gráfica INGEGRAF; 2002.

12. Orúe E, Laterra P, Cabria F. Expansión de la frontera agrícola en Argentina y erosión hídrica: mapas de riesgo utilizando el modelo USLE con apoyo de SIG. Procedente del Congreso de la Asociación Española de Teledetección; 2007 set 19-21; Mar del Plata, Argentina: Ed. Martin, 2007. p. $185-92$

13. Gallego FJ, Navarrete LJ, Montoro C, Vico J. Integración de la información relativa a la caracterización topográfica de la comarca olivarera "Sierra Mágina" (Jaén) en un SIG vectorial. Procedente de las Actas del XIV Congreso Internacional de Ingeniería; 2002 junio 5-7; Universidad de Cantabria, Santander: Gráfica INGEGRAF; 2002.

14. Nuñez J. Manejo y Conservación de Suelos. San José, Costa Rica: Editorial Universitaria Estatal a Distancia; 2001.

15. Almoza Y, Ruiz M, Medina H, Alonso G. Determinación de la erosividad de las precipitaciones en el oeste de Cuba. Zonas Áridas. 2007; 11(1): 139-49.

16. Alonso G, Ruiz M, Schiettecatte W, Díaz J, Almoza Y. Riesgo de erosión hídrica en la cuenca hidrográfica La Guira, Rio Cuyaguateje, Cuba. Zonas Áridas. 2007; 11(1): 110-123.

17. Golberg A. Síntesis de las ponencias presentadas en la primer jornada del taller sobre recuperación y manejo de ecosistemas naturales degradados. Procedente del Diálogo XLIX Recuperación y manejo de ecosistemas degradados, del Taller sobre recuperación y manejo de ecosistemas degradados; 1995 oct 24-26; La Pampa, Argentina. Montevideo: IICA-PROCISUR; 1998. p. 71-76.

18. Ministerio de Agricultura y Riego. El suelo y la cobertura vegetal. [Internet]. Lima: FAO; 2014. [Citado 15 enero 
2015]. Disponible en: http://agroaldia.minag.gob.pe/ biblioteca/download/pdf/manuales-boletines/suelos/ 2014/suelo_cobertura.pdf

19. Ministerio de Agricultura y Riego. Informe de la producción agropecuaria marzo 2014. Lima: FAO 2014. [Citado 15 enero 2015]. Disponible en: http://www. agroarequipa.gob.pe/sites/default/files/Marzo\%202014. pdf

20. Cárdenas G. La papa como ventaja competitiva. Pensamiento Crítico. 2009; 12: 55-78.
Manuscrito recibido el: 26/o1/15

Aceptado para su publicación el: 20/05/2015

\section{Correspondencia:}

Nombre: $\quad$ Eliana Contreras López

Dirección: Jr. Puno 1002 Jardín Botánico - Lima

e-mail: econtrerasl@unmsm.edu.pe 